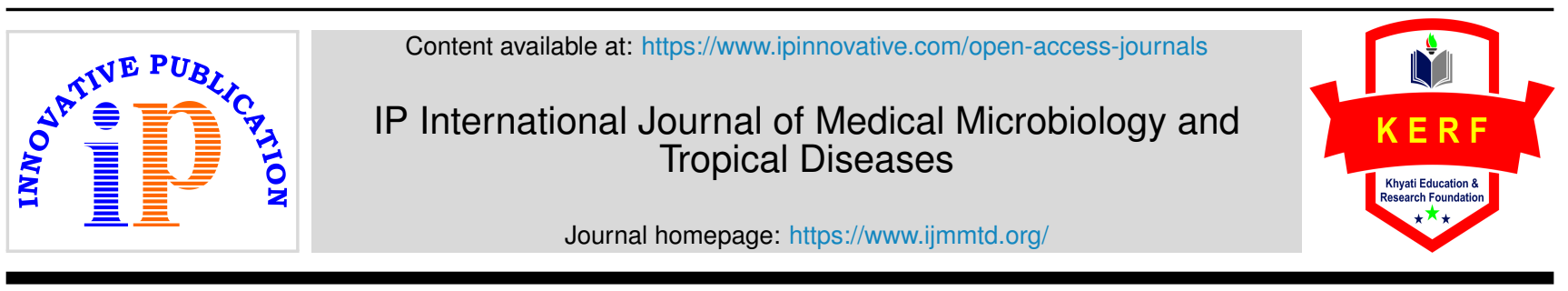

\title{
Editorial
}

\section{Challenges faced during COVID-19 pandemic}

\author{
Banur Raju Archana ${ }^{\circledR 1, *}$ \\ ${ }^{1}$ Dept. of Microbiology, Rajarajeswari Medical College and Hospital, Bengaluru, Karnataka, India
}

\section{A R T I C L E I N F O}

Article history:

Received 01-03-2021

Accepted 12-03-2021

Available online 26-03-2021
(C) This is an open access article distributed under the terms of the Creative Commons Attribution License (https://creativecommons.org/licenses/by/4.0/) which permits unrestricted use, distribution, and reproduction in any medium, provided the original author and source are credited.
An ongoing pandemic, Coronavirus disease (COVID-19) caused by Severe acute respiratory syndrome coronavirus 2 (SARS-CoV-2) has posed a global health threat in many countries and territories. A total of more than 78.6 Million cases of COVID 19 have been reported worldwide. In India about 10 Million confirmed cases and 1,46,000 deaths have been reported till now. COVID-19 has impacted every segment of life like economy, health care, education, sports, tourism, employment, entertainment, transport etc.

COVID 19 has resulted in significant decline in our economy. Major fall in the Gross Domestic Product (GDP) growth and stock market. Slowing of the manufacturing of essential goods, decrease in supply of essential products, losses in national and international business etc. The people from lower socio-economic status are greatly affected during the current pandemic due to economic decline. Nationwide lockdown has led to incur losses in small and medium enterprises market ratings project of over $\$ 4.5$ billion (₹35,000 crores) every day during the lockdown. The fourth-largest employer in the country is healthcare sector and specifically the private sector which provides nearly $80 \%$ of out-patient care and about $60 \%$ of in-patient care is currently facing $90 \%$ losses due to decreases in out-patient attendance, elective surgeries, and international patients. ${ }^{1}$

COVID19 has caused disruptions to health and health services in India in different ways. Initial challenges faced by healthcare workers is the diagnosis, contact tracing,

\footnotetext{
* Corresponding author.

E-mail address: drarchanasree@gmail.com (B. R. Archana).
}

quarantine, and treatment of suspected or confirmed cases of COVID 19. Despite of appropriate supplies, most hospitals faced huge shortage of beds, protective masks, sanitizers and even ventilator, ECMO machines for patients with severe cases. Demand for personal protection equipment has raised a hundredfold, leading to increase in price to several folds higher than regular price and delays in supply of medical items and diagnostic kits. There was a shortage of personal protective equipment worldwide, WHO warned that this will endanger the life of healthcare workers. Disruption of medical supplies and patients with other disease and health problems are getting neglected. There was a high burden on the functioning of the existing hospitals, overload on doctors and other healthcare professionals, who were at a very high risk. ${ }^{2}$

In addition to physical risks, the pandemic has placed extraordinary levels of psychological stress on healthcare workers exposed to high-demand settings for long hours, living in constant fear of disease exposure while separated from family and facing social stigmatization. Worry about bringing the virus home and passing it on to their family members - elderly parents, newborn and immunocompromised relatives. Healthcare professionals are under increased stress levels when handling uncooperative patients, not adhering to safety instructions, and feeling helpless when dealing with critically ill patients, as there is no definitive treatment available as well as limited availability of intensive care beds and resources. ${ }^{3}$ 
Pandemic has resulted in social disruption and mental trauma to the public. Even though the current crisis can bring with its opportunities for personal growth and family cohesion, disadvantages may outweigh these benefits. Disruption of celebration of cultural, religious, and festive events imposed a complete change to the psychosocial environment in affected countries. Isolation, contact restrictions, Social distancing with our peers and family members has resulted in undue anxiety, stress among the population. Added to that fear of contracting the virus led to the significant changes to our daily lives. Due to closure of schools, educational institutes, and online mode of education, affected mental health of children and adolescents significantly. Anxiety, lack of peer contact and depression were observed in children of all age groups. More counselling centres and/or helplines should be provided. Family counselling and additional support needs to be given to people with mental health disorders. Communities should be educated about mental health stigma and how to tackle mental health problems.

However, advances in research, will help to develop suitable therapeutic agents and vaccine against COVID-19 in a short time. Until then, we must rely exclusively on various preventive measures such as hand hygiene, social distancing, wearing mask etc. to control this pandemic. ${ }^{4}$

Similar to previous outbreaks, the current pandemic also will be contained shortly. This may not be the last pandemic we experience but the real question is, are we ready for the next pandemic /epidemic that may occur in the future. We must strengthen healthcare systems to include detection, containment, and treatment, as well as contact tracing and community mobilization. We must ensure the availability of well-trained and well-equipped global health surge capacity teams that can deploy to wherever outbreaks occur. Whether we will learn these lessons is yet to be seen. ${ }^{5}$

\section{Conflict of Interest}

None.

\section{References}

1. Tsamakis K, Rizos E, Manolis A, Chaidou S, Kympouropoulos $\mathrm{S}$, Spartalis E, et al. [Comment] COVID-19 pandemic and its impact on mental health of healthcare professionals. Exp Ther Med. 2020;19:3451-3. dor:0.38प2/etm.2020.8646.

2. Perez GIP, Abadi ATB. Ongoing Challenges Faced in the Global Control of COVID-19 Pandemic. Arch Med Res. 2020;51(6):574-6. doi:10.1016/j.arcmed.2020.04.016.

3. Lakhani A, Sharma E, Gupta E, Kapila S, Gupta S. Corona Virus (COVID-19) and its Impact on Health Care Workers. JAPI 2020;68:66-9.

4. Gopalan HS, Misra A. COVID-19 pandemic and challenges for socio-economic issues, healthcare and National Health Programs in India. Diabetes Metab Syndr: Clin Res Rev. 2020;14(5):757-9. do1:10.1016/].dsx.2020.05.041.

5. Fegert JM, Vitiello B, Plener PL, Clemens V. Challenges and burden of the Coronavirus 2019 (COVID-19) pandemic for child and adolescent mental health: a narrative review to highlight clinical and research needs in the acute phase and the long return to normality. Child Adolesc Psychiatry Ment Health . 2020;14(1):20. 101:0.1186/s 3034-(120$00329-3$

\section{Author biography}

Banur Raju Archana, Associate Professor (1) https://orcid.org/0000-00021854-3463

Cite this article: Archana BR. Challenges faced during COVID-19 pandemic. IP Int J Med Microbiol Trop Dis 2021;7(1):1-2. 